\title{
Intermédialités
}

Histoire et théorie des arts, des lettres et des techniques

Intermediality

History and Theory of the Arts, Literature and Technologies

\section{Déjouer : imaginaires de la fiction romanesque dans Les failles de l'Amérique et L'acquittement}

\section{Nicolas Xanthos}

Numéro 9, printemps 2007

Jouer

Playing

URI : https://id.erudit.org/iderudit/1005530ar

DOI : https://doi.org/10.7202/1005530ar

Aller au sommaire du numéro

Éditeur(s)

Centre de recherche sur l'intermédialité

ISSN

1705-8546 (imprimé)

1920-3136 (numérique)

Découvrir la revue

Citer cet article

Xanthos, N. (2007). Déjouer : imaginaires de la fiction romanesque dans Les

failles de l'Amérique et L'acquittement. Intermédialités / Intermediality, (9),

59-78. https://doi.org/10.7202/1005530ar
Résumé de l'article

Cet article s'intéresse à deux romans (Les failles de l'Amérique de Bertrand Gervais et L'acquittement de Gaétan Soucy) qui cherchent à déjouer l'activité lecturale en mettant sur pied des univers fictionnels qui ne semblent se déployer que pour mieux se désagréger. Par l'analyse de ces étranges dispositifs, on veut montrer que ces pratiques littéraires, qui donnent de prime abord l'impression de refuser le jeu fictionnel, finissent en bout de ligne par le réaffirmer en proposant de la fiction romanesque une conception qui met l'accent sur un principe de prolifération des univers et des individus, hors de toute nécessité de cohérence. Ces romans font ainsi une règle normative du jeu fictionnel; cependant, ils posent le principe de prolifération comme règle constitutive. Si ces romans « déjouent ", ce n'est donc pas sans enjeu. 


\title{
Déjouer : imaginaires de la fiction romanesque dans Les failles de l'Amérique et L'acquittement
}

\author{
Nicolas Xanthos
}

S

urtout s'il est de stratégie, le jeu ne va pas sans effet de surprise, sans art de la feinte. Constamment, il s'agit d'anticiper les gestes de l'autre, d'infléchir le cours de son action, de lui présenter des leurres comme des faits, de lui cacher le déploiement silencieux des armes qui auront raison de lui. Bien jouer, c'est déjouer l'autre.

Des figures du jeu de stratégie, l'art de la feinte ainsi conçu est sans doute l'une de celles qui rencontrent le plus fructueusement le terrain de la fiction littéraire, et de plus d'une façon. Art de la feinte, la fiction l'est ainsi assurément dans un premier sens évident, puisqu'il peut s'agir là de faire croire à ce qui n’a jamais eu lieu. L'évidence, toutefois, se trouble aux frontières de l'autofiction ou de la métafiction qui, du dehors ou du dedans, viennent brouiller l'espace de la vraisemblance. Art de la feinte, la fiction l'est aussi par exigence presque conversationnelle: comme Eco l'explique ${ }^{1}$, il ne vaut la peine de raconter que lorsque ce qui devait se produire n'a pas eu lieu. En principe, nul récit sans surprise. La fiction déploie ainsi événements et êtres sous les auspices de la précarité ${ }^{2}$, leurs états, désirs ou volontés étant appelés à être contrariés à plus ou moins brève échéance ${ }^{3}$. Art de la feinte, la fiction littéraire l'est encore en une troisième acception, plus retorse,

1. Lorsqu'il évoque les conditions élémentaires d'une séquence narrative. Voir Umberto Eco, Lector in fabula. Le rôle du lecteur ou la coopération interprétative dans les textes narratifs, trad. Myriem Bouzaher, Paris, Librairie générale française, coll. «Le Livre de Poche. Biblio essais », 1989 [1979], p. 137-138.

2. Il faut lire les commentaires éclairants qu'y consacre Richard St-Gelais dans Châteaux de pages. La fiction au risque de sa lecture, Montréal, Hurtubise HMH, 1994, p. 171-181 et p. 211-286.

3. Il est bien évident qu'on en reste ici à un degré de généralité qui ne rend pas justice à la multiplicité des avenues susceptibles d'être explorées par la fiction. On sait ainsi la place qu'ont prise dans la littérature contemporaine française le quotidien ou l'insignifiant qui, indépendamment d'un contenu dramatique de faible intensité, se caractérisent aussi par une narrativité sans surprise ou presque (à telle enseigne qu'il faudrait se demander si l'on a encore affaire à de la narrativité). 
plus fondamentale aussi: quand le texte, après avoir mis en place son univers diégétique, le détraque du dedans, s'emploie à en proposer des versions multiples et incompatibles, s'abîme en reflets variés et déformés d’un monde dès lors perdu.

Lorsque le roman investit cet espace incertain, on pourrait croire qu'il «refuse de jouer le jeu», pour reprendre l'expression de Grice dans un contexte analogue 4 : il semble pousser l'art de la feinte au-delà du terrain ordinairement balisé de l'usage fictionnel, inventer une pratique sans commune mesure avec les habitudes de lecture qui, dès lors, donnent l'impression de s'exercer en pure perte. Il ne joue plus avec nous, mais se joue de nous, serait-on tenté de penser. Et, à ce jeu-là, le lecteur se lasse rapidement.

À cette posture déceptive, nous voudrions en substituer une autre, confiante en le fair-play de ces textes qui vacillent. On fera ainsi l'hypothèse que ces dispositifs instables affirment en réalité un imaginaire de la fiction. Celui-ci inscrit en son centre le principe foisonnant de mise sur pied d'univers dont la permanence n'est jamais une propriété première et le bonheur frénétique d'une prolifération tous azimuts, sans égard pour une cohérence dont la compréhension lecturale fait pourtant son fil rouge.

Autrement dit, ces textes sont certes hors-jeu par rapport à une certaine manière de faire de la fiction, c'est-à-dire par rapport à une certaine réduction de l'espace des possibles fictionnels, mais ils le sont pour mieux dire une profonde adhésion à la fiction dans toute sa vitalité proliférante, pour mieux mettre en lumière, presque comme une revendication, ce qui fonde la possibilité même de l'espace fictionnel. Si donc ils déjouent la lecture, ce n'est pas pour se jouer d'elle; c'est tout différemment pour la ramener à l'essentiel, pour affirmer la nature première d'un territoire fictionnel qui demande à être reconnu pour ce qu'il est. En un dernier retournement, les feintes de la fiction par lesquelles les univers se volatilisent après s'être patiemment élaborés s'avèrent peut-être une manière d'exprimer ce qui, là, est réellement en jeu. Dans ces romans, nous voulons ainsi lire le déploiement d'un imaginaire, d'une pensée en acte, qui veut proclamer le propre de la fiction.

Deux romans nous permettront d'explorer ces hypothèses5: Les failles de l'Amérique de Bertrand Gervais ${ }^{6}$ et L'acquittement de Gaétan Soucy ${ }^{7}$. Ces deux

4. H. Paul Grice, «Logique et conversation», Communications, n 30, 1979, p. 64.

5. Un petit avertissement: les romans dont on va parler ici réservent plusieurs surprises. Au fil de notre réflexion, nous devrons en révéler quelques-unes, ce qui pourrait gâcher à l'avance certains plaisirs de première lecture.

6. Bertrand Gervais, Les failles de l'Amérique, Montréal, XYZ Éditeur, 2005. Désormais, les références à cet ouvrage seront indiquées par le sigle «FA», suivi de la page, et placées entre parenthèses dans le corps du texte.

7. Gaétan Soucy, Lacquittement, Montréal, Éditions du Boréal, 1997. Désormais, les références à cet ouvrage seront indiquées par le sigle «A», suivi de la page, et placées entre parenthèses dans le corps du texte. 
textes prennent les dehors de vues de l'esprit, d'expériences de (la) pensée. Ce sont, en effet, des psychismes déréglés qui y sont mis en scène: une conscience aux limites de l'hallucination permanente chez Gervais, une mémoire en constant renouvellement chez Soucy. Le plus raisonnable serait sans doute de postuler que ces univers fictionnels s'effondrent parce qu'ils mettent en scène des individus peu ou prou insensés, ou sont appréhendés par eux. Nous préférerons cependant déjouer, à notre tour, le sens commun et déplacer ce rapport pour supposer que l'instabilité cognitive des personnages est en vérité une «vraisemblabilisation » psychologique, de surface aurait-on dit en d'autres temps, qui vient justifier un enjeu fictionnel autrement fondamental, voire princeps. On veut donc croire ici que cette folie humaine est l'expression détournée d'une vérité de la fiction, au cœur de ce jeu.

\section{FICTION DE LA CONSCIENCE, CONSCIENCE DE LA FICTION :} LES FAILLES DE L'AMÉRIQUE DE BERTRAND GERVAIS

C'est en 2005 que Bertrand Gervais fait paraître cet imposant roman qu'est Les failles de l'Amérique. Le texte narre les étranges aventures de Thomas G. Cusson, étudiant québécois inscrit, à Santa Cruz, au doctorat en histoire de la conscience : son projet de thèse sur le Modulor qui s'étiole progressivement, ses difficultés d'enseignant, ses relations instables avec ses colocataires, son intérêt de plus en plus soutenu pour un tueur en série qui sévit sur les routes avoisinant Santa Cruz, les soupçons qui pèsent sur lui à la suite de plusieurs quiproquos, sa liaison intense avec la mystérieuse et (de plus en plus) distante Usha, ses problèmes informatiques qui prennent une importance et des proportions inattendues.

C'est à entrelacer ces diverses lignes d'intrigue que s'emploie ce roman, qui se présente pour l'essentiel ${ }^{8}$ comme le journal intime de Cusson, rédigé par ce dernier sur son ordinateur. Les chapitres, comprenant du reste parfois des dessins, se présentent comme autant de fichiers informatiques: ce support impose une structuration de l'expérience sur le mode de la fragmentation hypertextuelle, et, surtout, le rapport que Cusson entretient avec lui va devenir la mesure de son

8. «Pour l'essentiel», car chacun des chapitres commence par quelques pages consacrées d'abord aux tremblements de terre en général, puis aux tremblements de terre californiens et enfin, longuement, au tremblement de terre, californien toujours, du 17 octobre 1989 (date qui est aussi celle du dernier fichier informatique du journal de Cusson). À l'évidence, ces pages ne relèvent pas du journal intime. De plus, le roman commence par un bref récit qui narre le retour d'un personnage anonyme, après un tremblement de terre, sur les lieux où il a vécu, sa découverte dans les décombres d’un ordinateur qui s'avérera intact. Il faudra au lecteur traverser l'entier du roman pour comprendre que ce personnage est Cusson, et que ce premier événement du récit, au sens genettien, est en vérité le dernier de l'histoire. 
rapport au monde. Le mouvement diégétique d'ensemble du roman est apocalyptique: en effet, après s'être présentées sous un jour plutôt favorable, les choses vont de mal en pis. Les relations entre les personnages se dégradent. La vie académique de Cusson, comme chercheur et comme enseignant, va se compliquant: il se désintéresse petit à petit de son projet de thèse sans le remplacer pour autant, puis il est accusé d'agression sexuelle par une étudiante et doit suivre une thérapie. Sa relation de couple ne parvient pas à durer dès lors que son amoureuse prend ses distances. La plupart des gestes qu'il pose finissent par se retourner contre lui et le rendre suspect aux yeux des enquêteurs qui traquent le tueur en série dont on a parlé. Psychologiquement, enfin, Cusson devient de plus en plus instable et renfermé. Et rien ne vient entraver cette déchéance généralisée, qui se déploie sur un fond de violence larvée mais toujours perceptible.

À cette inéluctable dégradation de l'univers représenté répondent des perturbations de plus en plus importantes dans les moyens de la représentation. Pour une part, les balises chronologiques et référentielles du roman tendent à s'atténuer et le récit de Cusson émousse les arêtes des événements; graduellement, ces derniers se déploient non pas en tant que tels, mais dans leurs répercussions rêveuses à l'intérieur de la conscience du narrateur. Cette propension, qui gagne en puissance à mesure que l'on progresse vers la fin, était annoncée tôt:

Quand la fenêtre du traitement de texte s'ouvre, je n’hésite pas et je plonge, heureux de voir apparaître sans effort mes pensées, même les plus fugitives. Je ne fais qu'un avec la machine. L'Annexe disparaît, le monde se retire et seule persiste la lumière bleutée d'un écran qui me renvoie une image apaisante de mes rêveries. (FA, p. 43)

Métatextuelle à plus d'un titre, cette citation exprime les auspices sous lesquels se déploie l'activité narratrice: le retrait du monde et la rêverie. Et c'est en cela qu'on peut qualifier le roman d'histoire de la conscience, puisque ce sont la conscience, les modalités d'appréhension, voire de construction du monde qui sont mises de l'avant et non le monde en tant que tel - entre autres thèmes, bien entendu. L'espace consacré au musement' de Cusson croît - à mesure aussi qu'il coupe les ponts avec plusieurs personnages et se replie sur lui-même.

9. Par ce terme hérité de Peirce et Balat, on entend une activité de pensée proche de l'association libre ou de la rêverie. Bertrand Gervais dessine ses contours dans Lecture littéraire et exploration en littérature américaine (Montréal, XYZ Éditeur, coll. "Théorie et Littérature », 1998): «Le musement est l'imagination au travail» (p. 110); «un mouvement continu de la pensée, un flot qui nous traverse jusqu'à ce que nous nous déprenions de lui, pour une raison ou pour une autre» (p. 110); «[1] e musement est ce qui se trame en arrière-plan, pendant que le regard se perd et que l'attention flotte. Il est accès à l'aire enténébrée de la pensée, qui ne se laisse saisir que par des mouvements subits, des plongées dans le pur jeu des possibles» (p. 111). Il poursuit en indiquant que, sur le 
Pour une autre part, et c'est ce qui nous intéressera maintenant, de sérieuses incompatibilités fictionnelles apparaissent. En ces lieux instables, la fiction semble vouloir déjouer l'activité lecturale, minant après-coup la représentation qu'on s'est construite du monde décrit. On prendra comme premier exemple de ce phénomène une scène que Cusson surprend chez une de ses camarades, Rikki, qui le soupçonne d'être l'auteur des violences physiques qu'a subies Kama, un autre étudiant du doctorat. Cusson se rend chez Rikki pour essayer de plaider sa cause; arrivé à destination, il ne frappe pas à la porte d'entrée mais passe par derrière et surprend un souper entre Rikki, Kama, son directeur de thèse Forsythe et un professeur français invité, Maçon. Il commence par s'inquiéter des conversations :

Que se disaient-ils entre deux bouchées de pâtes fraîches? Je ne pouvais le saisir. On riait allégrement et j'entendais résonner mon nom jusque sous les cèdres. J'étais la risée. Et je me transformerais bientôt en bouc émissaire qu'on traînerait dans la mer avant de le sacrifier sur l'autel de la démesure. (FA, p. 399)

Rapidement, la scène prend une tournure imprévue :

Je devais à tout instant retenir ma respiration. Je me serais cru dans l'alcôve, au plus fort de ma relation avec Usha, quand nous rêvions de couples qui s'exécutaient sur le futon déplié de l'Annexe. [...] Déjà le chemisier de Rikki était ouvert, offrant à Forsythe des seins que peu d'hommes avant lui avaient pu toucher. Kama avait relevé ses manches et s'était agenouillé devant [Maçon] qui contemplait son verre d'un air absent. Forsythe plongeait sa main profondément dans le pantalon détaché de son étudiante, Maçon tâtait à l'aveugle les chairs gonflées de son protégé. Les chaises du salon se transformaient en accessoires obscènes. Kama était à genou sur le siège tandis que Maçon lui faisait une fellation, serrant de ses deux mains le postérieur musclé de son poulain. Forsythe et Rikki s'embrassaient tandis qu'elle s'assoyait lourdement sur son sexe bandé. (FA, P. 400)

De façon à disposer d'une monnaie d'échange pour consolider une position académique menacée, Cusson décide de prendre des photographies incriminantes des convives. Il va chercher son appareil dans son véhicule et revient en toute hâte - pour constater ce changement:

[...] les lumières de l'appartement de Rikki étaient éteintes. On n'y voyait plus rien. Les chandelles avaient été soufflées, la table, nettoyée et les chaises, rangées.

plan de la technique romanesque, «les exemples de monologue intérieur ou "stream of consciousness" [...] visent bel et bien une mise en récit du musement, par la voie d'une conscience reproduite à même ses mouvements et dont rien ne semble contraindre la logique associative.» (p. 111) 
Où étaient-ils tous partis? Où s'étaient sauvés ces corps trempés de sueur et de sécrétions? Il ne restait plus rien. Les bouteilles de vin avaient disparu, les assiettes salies, les vêtements emmêlés des convives. J'ai collé mon visage contre la moustiquaire. Le salon était désert. [...] Je suis resté immobile devant la moustiquaire, les jambes écartées, un bras levé bien haut. Je ne savais plus où j’étais ni sur quel plan se déroulaient les événements qui m’impliquaient. Je faisais le mort dans le cadre de la porte.

Rikki a hurlé. Elle portait un vieux t-shirt de Duane que son cri a déchiré. (FA, p. 400-401).

La surprise du personnage répond à celle du lecteur, déjoué par cette séquence qui met en scène chez Rikki un petit univers pour mieux ensuite en effacer les moindres constituants: à la lumière s'est substituée l'obscurité; au bruit, le silence; aux quatre personnages joyeux puis dépravés, un salon désert puis un seul personnage, inquiet; au chemisier ouvert, un vieux t-shirt déchiré. La rapidité avec laquelle Cusson va chercher son appareil photo et revient empêche aussi de supposer à cet effacement généralisé une cause actorielle : le temps aurait manqué aux quatre convives pour tout remettre ainsi en ordre. Ce qui s'est produit est, littéralement, incommensurable - ce dont témoigne la position de Cusson (jambes écartées et un bras levé), qui reproduit le Modulor, impossible mesure.

Le rapport de Cusson à l'écriture informatisée semble s'être généralisé, et devenir le principe de son rapport au monde. On se rappellera en effet que, dans un extrait cité plus haut, Cusson écrivait sur le mode de la rêverie; ce mode provoquait la disparition de l'Annexe et le retrait du monde. Désormais, ce n'est plus seulement cette relation médiatisée, scripturale, du monde qui procède de la rêverie, mais bien, aussi, la perception du monde elle-même. Le musement ne se limite plus à l'écriture, mais s'étend à l'appréhension du monde; ce dernier est dès lors toujours fonction d'un imaginaire susceptible de l'investir, de le structurer, de s'y déployer. Cusson lui-même semble le suggérer, en un propos à double entente: "J'avais droit à une scène comme j'avais tant rêvé d'en voir, une scène spontanée et pornographique, faite d'un abandon licencieux à la limite de l'intolérable» (FA, p. 400, nous soulignons). Cette scène est, littéralement, le rêve devenu réalité.

La conséquence implicite de cette feinte de la fiction, qui donne à lire pour réel ce qui n'est qu'imaginaire ${ }^{10}$, est un vaste soupçon lectural: si cette scène «spontanée et pornographique» est fictive du fait d'une invasion de l'imaginaire, ne peut-il pas en aller de même pour d'autres scènes romanesques, voire pour l'entier de la représentation? Cette scène est-elle exceptionnelle parce que

10. On nuancera dans un instant cette dichotomie qui ne rend pas pleinement compte de ce qui se passe dans le roman. 
Cusson hallucine ou parce que, pour une fois, la coprésence du réel et de l'imaginaire nous permet de savoir avec certitude qu'il hallucine, alors que d'ordinaire nous ne le savons pas?

L'étendue effective du soupçon lectural provoqué par ce manquement au jeu de la représentation fictionnelle dépend somme toute du reste du roman : s'il s'agit d'un épisode unique, on poursuivra en prenant pour argent comptant les propos du narrateur; si par contre l'instabilité ici mise en scène est confirmée par d'autres portions du texte, c'est le tout du monde représenté qui risque de faire les frais d'une suspicion réactivée. On s'en doute, le roman déjouera encore, et radicalement cette fois.

Tout au long du texte, le narrateur vit une relation trouble avec une jeune femme prénommée Usha. Il la rencontre dans le jardin botanique de Kresge College (FA, p. 83-85). Il l'y retrouve pour un premier baiser et un rendez-vous, quelques jours plus tard, dans une librairie (FA, p. 92-94). Il la revoit dans cette librairie où elle fait la lecture publique d'un texte érotique qui reprend certains éléments de leur début de relation, ce qui heurte Cusson, et ils font l'amour dans la voiture de ce dernier (FA, p. 104-109). Elle lui donne quelques jours plus tard un extrait du recueil de nouvelles érotiques auquel elle travaille et intitulé Traité des pratiques sexuelles, qui reprend des portions de l'intimité de Cusson que ce dernier n'avait pourtant pas évoqué à Usha (FA, p. 120-123). À partir de là, Cusson décrit à plusieurs reprises leurs rencontres, qui prennent un tour d'exploration sexuelle toujours plus intense fournissant de la matière aux fictions d'Usha mais éloignant progressivement les deux amants (FA, p. 134-138; 163-166; 234-238; 289290; 303-307); ces rencontres ont lieu au motel des parents d'Usha, le Indian Inn, où elle dispose d'une chambre, ou chez Cusson. Ils se retrouvent enfin au jardin botanique pour constater la mort de l'arbre devant lequel leur relation avait commencé, ce qui fait dire à Usha: «Ce qui nous liait n’existe plus » (FA, p. 409). Cette rupture ainsi officialisée, Thomas et Usha ne se reverront plus.

Cusson fait tout pour garder cette relation secrète. Toutefois, lorsqu'il est sérieusement soupçonné de jouer un rôle dans les meurtres en série commis autour de Santa Cruz, il invite finalement les enquêteurs qui l'interrogent à aller vérifier ses protestations d'innocence auprès d'Usha:

La seule qui pourra tout vous confirmer, la seule qui vous dira que je ne suis pas le tueur que vous cherchez, c'est Usha. Usha Patel. Là encore, c'est compliqué. Ses parents ne savent pas que nous nous sommes fréquentés tout l'hiver et une bonne partie de l'été. Il se peut qu'elle ne veuille pas parler devant eux, mais prenez-la à l'écart et elle vous dira tout. (FA, P. 435)

Les deux enquêteurs, Frost et Hoover, vont procéder aux vérifications et reviennent avec ces nouvelles: 
On a parlé à monsieur et madame Patel. Séparément. Il y a un problème. Un problème de taille.

Qu'est-ce qu'il y a? Ils ont refusé que vous parliez à Usha?

C'est plus gros que ça, complète Frost. Ils n'ont pas de fille. Pas d'enfant.

On a vérifié dans les registres. Ils n'ont jamais déclaré d'enfants. On a même demandé à des parents et à des amis. Ils n'ont jamais eu d'enfant. Madame Patel est stérile.

Usha n'existe pas.

Tout est fini. [...]

J'ai tout imaginé depuis le début; il n’y a pas d'autre explication. (FA, p. 437)

Si les conséquences lecturales du souper chez Rikki pouvaient encore être limitées à l'événement en question, il en va tout différemment avec cette nouvelle feinte fictionnelle. On l'a vu, la relation de Cusson et d'Usha occupe un vaste espace romanesque: quantitativement autant que qualitativement, il s'agit d'une ligne d'intrigue de première importance, dont les ramifications dans l'existence du narrateur sont innombrables. L'importance qualitative d'Usha se marque encore en ceci que, d'une part, Cusson se fonde uniquement sur elle pour prouver son innocence, et, d'autre part, les procédures de vérifications sont l'œuvre de ces spécialistes que sont les enquêteurs. On ne peut dès lors procéder ainsi à l'effacement d'Usha sans atteindre les fondements de l'activité narratoriale, c'està-dire les fondements de la «réalité » de tout l'univers mis en scène ${ }^{11}$.

À en rester à ce sentiment d'apparente tricherie (on a donné pour réel ce qui n'était qu'imaginaire, et on lève tardivement le voile sur ce mystère entretenu à coups d'informations tronquées), on manquerait toutefois la pensée autrement complexe de ce dispositif fictionnel subtil, dont deux traits demandent à être mis en lumière. D'abord, les scènes avec Usha fourmillent de phrases à double entente qui fonctionnent comme autant d'avertissements invisibles à la première lecture. La première rencontre est introduite comme suit par Thomas qui, assis sur un banc, venait de dessiner le Modulor et l'homme de Vitruve et les comparait:

Je devais me ressaisir, imaginer quelque chose de neuf.

Tandis que je complétais mes dessins, une fille s'est assise sur le banc voisin. (FA, p. 83, nous soulignons)

11. L'effet de ce doute rappelle d'autres doutes que Wittgenstein évoque dans De la certitude: «613. [...] Mais qu'est-ce qui pourrait bien me faire douter si cet homme est bien N. que je connais depuis tant d'années? Ici, un doute semblerait tout emporter avec lui et tout précipiter dans le chaos. 614. C'est-à-dire: Si j'étais en butte à des contradictions venant de toute part (qu'on me dise que cet homme ne s'appelle pas comme je l'ai toujours su - et j'emploie ici "su” à dessein), dans ce cas ce seraient les fondations de toute mon activité de jugement qui se déroberaient sous moi.» (Ludwig Wittgenstein, De la certitude, trad. Jacques Fauve, Paris, Éditions Gallimard, coll. «Tel», 1995 [1951], p. 141) 
À la suite de leur deuxième rencontre, Cusson décrit son état: «Étais-je heureux? En état d'ébriété, oui. J'étais prêt à réinventer le monde» (FA, p. 93. nous soulignons). Pendant la lecture publique, il observe Usha: «L'avais-je véritablement embrassée dans la Volvo? Elle et moi ne semblions pas appartenir au même univers » (FA, p. 105 - nous soulignons). Elle lui remet, plus tard, un extrait du Traité et il constate: "Je vais devoir m’habituer à sa manie de tout écrire, de faire de la fiction avec tout, jusqu'à notre vie sexuelle» (FA, p. 120 - nous soulignons). Il remarque aussi que le texte comprend des éléments qu'il avait cachés à Usha: «Le plus étrange est que je n'avais pas tout raconté à Usha. J'avais omis certains détails. Pourtant, je les retrouvais là, dans le Traité, comme si je l'avais écrit moimême» (FA, p. 122). Il pense plus loin à sa compagne: «La femme est le mystère de l'homme... Usha était à ce titre une merveille faite chair. Elle échappait aux lois du quotidien. Son corps était un songe» (FA, p. 138, nous soulignons). Durant leurs ébats, il la prend en photo et veut partir avec les polaroïds, ce qu'elle refuse catégoriquement: «Elle ne s'en départirait jamais. Elle m'a fait comprendre que ces polaroïds n'existaient pas. Cette séance de photos n'avait jamais eu lieu. J'avais tout imaginé» (FA, p. 165, nous soulignons). Il écrit seul les «dix vérités d'Usha », au nombre desquelles on compte celle-ci : «Mes pulsions, mes désirs et mes craintes, mes souvenirs enfouis, mes fuites oubliées, mes mains, mon sexe, mes trous, tu es tout ça» (FA, p. 248). Il évoque ses peurs: "peur que tu ne sois plus qu'une pâte à modeler conçue pour assouvir mes désirs, ces choses qui me poussent dans la tête et que je ne parviens pas à contrôler, [...] des désirs qui étendent leur emprise sur le reste de mes fonctions» (FA, p. 290).

Ainsi, dès la toute première rencontre, et continûment ensuite, les scènes qui réunissent Cusson et Usha sont serties de ces phrases dont la double signification pragmatique omniprésente manifeste discrètement la consubstantialité du réel et de l'imaginaire (ou du fantasme, car on a dit le nombre de scènes érotiques décrites) - ou encore d'un monde factuel unique et de ses variations imaginatives qui le constituent tout autant. La conscience du monde de Cusson ne se distingue pas de son musement - ou plus exactement comprend nécessairement son musement. Voir le monde, c'est y mettre du sien. C'est dire, aussi, que ce roman, en son principe, est à l'opposé d'un objectivisme fondé sur une dichotomie réel versus imaginaire et postule plutôt la double implication réel alors imaginaire, et réciproquement. Histoire, ou fiction, de la conscience, donc, que Les failles de l'Amérique.

Il faut encore mettre en lumière un second aspect de la pensée manifestée par ce dispositif. Avec patience et par petites touches, le roman construit en effet une équivalence entre l'activité de la conscience telle qu'on vient de la décrire et la fiction. On a déjà indiqué, à ce chapitre, que le rapport au monde de Cusson présente de nettes similitudes avec son rapport à l'écriture. On peut maintenant faire quelques pas de plus. 
Dans la scène du souper chez Rikki, alors que le repas prend un tour pornographique, Cusson écrit: "Le dernier chapitre du Traité s'écrivait sous mes yeux et je n'avais rien pour prendre des notes» (FA, p. 399, nous soulignons). Il faut reprendre certains éléments du récit de la liaison avec Usha pour voir la part qu'y prend l'activité scripturale d'abord, puis plus spécifiquement la fiction. Lors de leur deuxième rencontre, Cusson retrouve Usha un stylo à la main, en train d'écrire dans un cahier; puis elle l'invite, comme on l'a dit, à une lecture publique dans une librairie. Le récit érotique qu'elle lit alors reprend la scène de la première rencontre entre les deux personnages. Cusson en est un peu vexé et elle lui répond, avec une ironie involontaire qu'on saisit à la seconde lecture :

Il ne fallait pas confondre la vie et la fiction, m’a-t-elle sermonné. Ce n’est pas moi dans son récit, mais un personnage. Une fiction. D’ailleurs, il s'appelait Gabriel et non Thomas. [...] Je devais faire la part des choses. Ce qu'elle pouvait vivre était peut-être à l'origine de ses écrits, mais rien n'y était transposé directement. [...] Ce n'étaient que des mots, une fiction qui ne pouvait menacer notre vie ou nos souvenirs. Gabriel était une fiction. Il ne pouvait rien détruire. C'était un être de papier. Une fiction créée à l'ordinateur. (FA, p. 108-109)

Le Traité va progressivement prendre cette tournure dans le roman: une œuvre de fiction qui décrit la réalité de la relation entre Usha et Cusson, s'en nourrit, la nourrit. Un principe d'interpénétration s'instaure entre fiction et réel - comme, tout à l'heure, ce principe s'instaurait entre conscience (c'està-dire, entre autres, imaginaire et fantasme) et réel. Imaginaire et fantasme se font dès lors, par le biais des nouvelles érotiques d'Usha, le terrain partagé de la conscience et de la fiction - qui, par rebond, en viennent à s'équivaloir. En d'autres mots, ce qui est dit de la fiction vaut pour la conscience, et ce qui est dit de la conscience vaut pour la fiction; chacune devient mode d'expression de l'autre.

La fiction est ainsi manière d'élaborer des mondes en marge d'un factuel monolithique, prolifération de signes travaillés par toutes les formes ou les déformations de l'imaginaire, les réfractions du désir et de la peur, la complexité infinie d'une subjectivité en mouvement et en musement. Elle est (di)version du monde, brouillage des ordres, logique du foisonnement, métamorphoses continues. Et c'est vers cette pensée de la fiction que pointe le dispositif complexe des Failles de l'Amérique - qui devient, en un nouveau détour, conscience de la fiction.

C'est ainsi que, si le lecteur peut certes éprouver l'impression d'être déjoué par le roman, il peut certes voir ses promenades inférentielles ${ }^{12}$ apparemment

12. Selon l'expression d'Eco qui désigne par là les diverses suppositions que le lecteur est amené à faire au fil de sa lecture. Umberto Eco, Lector in fabula, p. 142-156. 
contredites par l'univers représenté, il n'en reste pas moins que la fiction, ici, ne se borne pas à décevoir. Elle ne respecte pas les règles de cohérence de la représentation, mais pour cette bonne raison qu'ici, ces règles ne disent pas le fondement de la fiction. Elles en domestiquent plutôt l'usage, le réduisent à un fonctionnement convergent, l'amputent de sa vitalité première. Le roman refuse ces contraintes pour mieux en dire la normativité et exhibe plutôt, nues, les conditions de possibilité de la fiction. Il se retire d'un jeu dont il veut par ce geste montrer la nature accessoire - pour mieux se déployer sur un terrain essentiel. Ce faisant, il invite le lecteur à le rejoindre sur ce terrain inhabituel, cet « endofictionnel ${ }^{13}$ » où la fiction s'ouvre comme aire discursive.

\section{NON-LIEU DE MÉMOIRE : L'ACQUITTEMENT DE GAÉTAN SOUCY}

Ce court roman paru en 1997 est le deuxième texte publié par Gaétan Soucy. À la différence d'autres œuvres de l'auteur, à la configuration narrative complexe, L'acquittement propose une ligne d'intrigue plutôt épurée: Louis Bapaume revient dans le village où il avait travaillé, vingt ans auparavant, comme professeur de musique; les raisons de ce retour sont longuement tues dans le roman, mais on finit par apprendre que Bapaume vient y demander pardon à une jeune femme, Julia von Croft, de l'avoir quelque peu maltraitée quand il lui donnait des leçons de piano, vingt ans plus tôt. Cette ligne d'intrigue se complique de deux manières. D’une part, les von Croft (Robert, Maurice, son fils, Julia et Geneviève - deux jumelles) ont décidé de taquiner Bapaume et lui présentent Geneviève en la faisant passer pour Julia, et vice-versa; d'autre part, les allusions à la vie familiale présente et passée de Bapaume se multiplient au fil du texte, mais en livrant des images qui ne s'avèrent pas toujours pleinement compatibles. Se construit ainsi une fiction où, sur plusieurs plans et de plusieurs façons, l'on se joue et se fait jouer des tours plus ou moins délibérés - jusqu'aux deux dernières lignes du texte qui jouent, cette fois-ci au lecteur, un tour ultime et décisif, renversant tout ou presque de ce que le roman avait érigé pendant près de cent dix pages.

Avec constance et dans divers registres, le roman travaille cette idée d'une mystification. Ainsi, le lendemain de son arrivée, Bapaume fait le trajet de la gare à Saint-Aldor dans un traîneau à chiens guidé par Maurice von Croft, le frère des deux jumelles. L'homme éprouve une réelle affection pour l'adolescent mais ne sait comment l'aborder: «Ils étaient deux enfants timides qui sympathisent de

13. Néologisme forgé sur le modèle de l'«endonarratif». 
loin au terrain de jeu et n'osent le premier signe de reconnaissance» (A, P. 51). Il souhaite qu'ils puissent fraterniser :

Il y avait un thermos calé au fond du traîneau. Louis le supposa rempli de café chaud. En boire assis à l'ombre d'un rocher, sans penser à rien, ensemble et seuls au milieu de la neige, abrités sous la même couverture, entourés d'un silence qui serait comme la musique de cette blancheur, ce n'aurait pas été un crime. Il aurait pu même, qui sait? prendre la main de Maurice dans la sienne...

Comme si son vœu s'exauçait, le garçon stoppa l'attelage au milieu d'une clairière. Il marcha jusqu'à l'orée du bois. Il écarta les jambes.

- Retournez-vous! lança-t-il par-dessus son épaule.

Louis rougit. Il entendit le tambourinement du liquide chaud trouant la croûte gelée. (A, P. 52)

La mystification demeure strictement actorielle, et de l'ordre de la mésinterprétation. Les faits s'avèrent tout différents de ce que Bapaume avait espéré ou imaginé, sur la base de quelques regards échangés: Maurice von Croft arrête le traîneau non pas pour que lui et Bapaume puissent partager un moment en commun, mais pour qu'il puisse se soulager. À la relative grâce de ce que Bapaume avait espéré se substitue la trivialité d'un geste bien différent. Et toute la scène est construite sur le principe de cette opposition : à la proximité souhaitée se substitue la distance; à la position assise en commun, la position debout séparés; au silence, le tambourinement; au liquide ingurgité, le liquide expulsée ${ }^{14}$.

Une partition progressivement découverte par Bapaume donne aussi lieu à une semblable mystification. Bapaume en voit une première partie dans les feuilles de musique de Maurice et il en est renversé:

Louis regarda devant lui, abasourdi. Voilà donc à quoi se consacrait le jeune Maurice !... Il lut de nouveau, n'en croyant pas son cœur. La retranscription elle-même, les signes de musique avaient été tracés d'une main sûre, aguerrie, avec une netteté géométrique, le résultat avait le caractère étincelant d'un manuscrit de Mozart. Et les annotations fines, intelligentes, manifestaient une compréhension réelle de cette merveille de contrepoint. (A, p. 64)

En parlant plus tard avec Maurice, il apprend que cette partition est l'œuvre non pas de l'adolescent, mais d'un de ses enseignants, le frère Decelles. Plus tard encore, il tombe sur le reste de la partition, dont la beauté l'émeut toujours autant: «Le finale était constitué d'une prière du Pardon. Celle-ci allait bien au-

14. Cette scène trouvera d'ailleurs un écho identiquement construit dans les derniers moments que Bapaume partage avec Maurice, avant de rallier la gare (A, p. 108-110). Un semblable principe d'opposition régit du reste la scène du souper pornographique chez Rikki dans le roman de Gervais. 
delà de la simple maîtrise du contrepoint, elle touchait à la grâce, à la pureté du déchirement, et Bapaume sentit des larmes lui piquer les paupières » (A, p. 77). Il ne peut s'empêcher d'imaginer le frère Decelles:

Oui, le frère Decelles aussi devait éprouver le sentiment d'être enterré vivant dans le cercueil de l'oubli, dans la terre grasse de l'indifférence des hommes et de l'avenir. Et persévérer quand même, lorsqu'on sait qu'il est impossible de réveiller les morts, continuer envers et contre tous les harassements de l'invention représentait un entêtement et un courage dont les gens qui ne créent rien, ces cadavres ambulants, n’avaient pas idée. "J'écrirai à ce frère Decelles ", se dit Bapaume. (A, p. 77, nous soulignons).

Ladverbe «aussi » montre bien tout ce que cette représentation que Bapaume se fait du frère Decelles doit à son image de lui-même. Cet autoportrait d'un inconnu, si l'on ose dire, exprime également la manière dont Bapaume appréhende les êtres: à l'aune de lui-même. Lorsqu'il pense à Maurice ou au frère Decelles, c'est dans le fond à lui-même qu'il pense, c'est lui-même qu'il voit en eux. Si le processus projectif n'est pas nouveau, le roman prend par contre grand soin de construire ses scènes pour que la nature projective des inférences sur l'être d'autrui apparaisse avec netteté - précisément grâce à la présence de la mystification rapidement démystifiée.

Mais l'aventure de la partition ne s'arrête pas là, puisqu'une ultime surprise attend Bapaume et le lecteur à la fin du manuscrit:

Brusquement [Bapaume] jeta la feuille sur la table, du même mouvement hagard dont il se serait dessaisi d'un couteau ensanglanté. Au bas de la page, une notice écrite en caractères si petits qu'il fallait s'y coller le nez pour la lire:

Retranscrit des archives de Saint-Aldor par le frère Adrien Decelles. Composé autour de 1927 par M. Louis Bapaume, maître de musique à l'orphelinat de la Crucifixion. (A, p. 78 ).

La feinte, ici, ne se limite pas au domaine diégétique, malgré la stupéfaction de Bapaume: c'est l'activité lecturale elle-même qui finit par être perturbée à force de devoir reconfigurer l'univers décrit. La première apparition du manuscrit - dont Maurice serait l'auteur - semble orienter les inférences vers le scénario du jeune surdoué qui ne paie pas de mine; la deuxième - l'auteur est cette foisci le frère Decelles - pointe plutôt, comme on l'a dit, vers la nature projective de la compréhension d'autrui; la troisième - Bapaume est le véritable auteur de la partition - ouvre sur une question sans réponse : comment Bapaume, ce musicien pour qui la création a une importance déterminante, a-t-il pu oublier qu'il a été l'auteur de cette œuvre d'une qualité visiblement supérieure? Comment oublier cela? S'il peut oublier cela, de quoi peut-il - et pouvons-nous - ensuite être sûr? 
Si Les failles de l'Amérique se constitue autour des troubles d'une conscience, et donc du rapport au présent, à l'immédiat, Lacquittement s'élabore autour des troubles d'une mémoire, et donc du rapport - en grande partie identitaire - au passé. C'est là que de très sérieuses perturbations fictionnelles prennent leur source, qui vont jusqu'à mettre en péril l'entier de la représentation romanesque. Les glissements autour de la partition, qui ne sont pourtant pas sans poids, apparaîtront finalement mineurs par rapport à deux éléments mémoriels dont on voudrait parler maintenant.

Bapaume, on l'a dit, est venu demander pardon à Julia von Croft. La veille de la rencontre, un personnage secondaire, Chouinard, lui montre un objet qu'un harfang avait laissé tomber de ses serres: "C'était un jouet pour enfants: un ourson de peluche. Le ventre en avait été déchiré, sans doute par le bec et les serres du rapace. Il lui manquait un œil. Une des oreilles avait été arrachée » (A, p. 33). Ledit Chouinard se met en devoir de le rapiécer, et Bapaume constate la transformation le lendemain matin: «L'ourson de la veille avait été reprisé [...]. Chouinard lui avait cousu un bouton de culotte à la place de l'œil droit, mais une oreille lui manquait toujours » (A, p. 49). La rencontre de Bapaume et de Julia ${ }^{15}$ se déroule sur plusieurs pages au long desquelles le musicien apparaît comme réellement affecté; il explique notamment à Julia que l'idée de lui demander pardon s'est imposée à lui à la mort de son fils: il n'aurait jamais accepté que qui que ce soit lui fasse subir ce que lui, Bapaume, a fait subir à Julia. Les allusions à l'état psychologique du personnage sont nombreuses durant le dialogue, et la douleur de Bapaume est durablement indiquée. Arrive le cœur dramatique de cette scène, juste après que Bapaume a expliqué à Julia les motivations de son geste:

Il ouvrit son bagage avec des gestes empreints de tendresse et de tranquillité.

- J'aimerais que vous acceptiez ceci, Julia.

Julia ramena frileusement ses mains sur sa poitrine, comme si elle n'osait toucher à ce qu'on lui offrait. C'était un ourson de peluche. Le ventre avait été raccommodé avec du gros fil. Il avait un bouton de culotte cousu à la place de l'œil droit, et une oreille arrachée.

- C'est l'ourson que mon fils avait quand il était tout petit. Il avait tellement joué avec lui que, voyez, le ventre en avait été déchiré, il avait perdu un œil, une oreille.

15. Pour ne pas rendre plus compliquée encore une situation déjà bien embrouillée, nous parlerons de l'interlocutrice de Bapaume dans cette scène comme de Julia von Croft - c'est du moins ce que croit Bapaume et ce que le lecteur croit aussi, compte tenu des informations dont il dispose à ce moment. Plus tard, on apprendra qu'il s'agissait en fait de sa sœur jumelle Geneviève - le tout étant une plaisanterie mystificatrice faite à Bapaume et dont tous les membres de la famille von Croft étaient complices. 
Il l'avait reprisé lui-même, je me rappelle; il m’avait piqué un bouton de culotte... Accordez-moi, Julia, la faveur de l'accepter.

- Mais, monsieur Bapaume, jamais je n’oserais...

- Acceptez-le. Ainsi, et ainsi seulement, je pourrai croire que vous m'avez pardonné.

Julia l'interrogea des yeux. Puis elle approuva gravement. Elle venait de comprendre le sens de ce don. Elle cala l'ourson contre son sein. (A, p. 103-104)

Dissipant les derniers doutes que le lecteur pouvait nourrir, Chouinard, peu après le départ de Bapaume, constate la disparition de l'ourson : «L'ourson! disait Chouinard dans tous ses états. Où est-il? Je l'avais laissé ici ce matin, sur le comptoir, j’en suis sûr! » (A, p. 119)

Comme le titre du roman le dit, cette scène où Bapaume demande pardon à Julia est le cœur de l'édifice : c'est ce qui explique la venue de Bapaume, ses sentiments souvent malheureux fréquemment mentionnés; c'est là que convergent en somme tous les éléments narratifs et thématiques du texte. Aucun des commentaires psychologiques du narrateur durant cette scène ne laisse supposer que Bapaume ment ou cherche à manipuler délibérément Julia. Plus encore, dans le passage cité, le narrateur ne fait aucunement mention à une feinte volontaire de Bapaume: l'ourson est certes décrit en des termes identiques à ceux de sa première description, mais l'identité des deux objets n'est pas affirmée ni glosée; de plus, lorsque Julia saisit la signification du geste de Bapaume, aucune modalisation n’apparaît, qui laisserait filtrer une évaluation narratoriale critique de la scène.

L'ourson apparaît comme totalement dissonant dans le passage; et la même question qu'on a souvent posée revient ici : si un élément fictionnel aussi manifestement problématique peut apparaître à ce moment crucial, de quoi peut-on ensuite encore être sûr? Faut-il considérer que Bapaume ment délibérément à Julia? En principe, l'hypothèse est plausible, mais aucune information textuelle, comme on l'a vu, ne vient l'appuyer. Doit-on estimer que Bapaume croit réellement que cet ourson est celui de son fils, quand bien même il l'a vu pour la première fois la veille? Si tout plaide en ce sens, on voit mal l'univers mental que cette hypothèse présupposerait.

Une incertitude sérieuse pèse désormais sur Bapaume, que le roman n'éclaire pas. La portée exacte du coup qui vient d'être ainsi porté à la représentation romanesque semble toutefois difficile à déterminer. Le lecteur peut très bien limiter les dégâts en bornant l'effet de la remise en question à un mystère psychologique localisé : au plus fort de la scène du pardon, Bapaume a posé un geste incompréhensible. Cela ne met ainsi pas en péril le reste de l'univers diégétique. Il n'en ira toutefois pas de même avec le second événement problématique qu'on voudrait décrire maintenant. 
Au cours des vingt-quatre heures que dure l'histoire de L'acquittement, on apprend, par petites touches, la vie familiale présente et passée de Bapaume. Le premier soir, ne pouvant se rendre à Saint-Aldor, Bapaume est hébergé par le lieutenant Hurtubise. Bapaume lui apprend que son épouse s'appelle Françoise et est juive d'origine parisienne et violoniste - tout comme la mère de Hurtubise, dont un portrait trône dans le logis du lieutenant. Plus tard, seul dans sa chambre, Bapaume trouve dans son bagage une lettre de Françoise, qui lui reproche d'avoir abandonné son oratorio, dont elle a pourtant besoin pour exister, qui lui reproche aussi sa mission chez les von Croft et le conjure de rentrer rapidement auprès d'elle, car le temps presse: "Mes doigts sont glacés. Il y a du frimas aux fenêtres. Je ne sens presque plus battre mon cœur dans ma poitrine» (A, p. 41). Le lendemain, chez les von Croft, Bapaume écrit une réponse à Françoise, mentionnant d'emblée son fils, dans un contexte qui pourrait laisser croire à sa disparition: "Comme tu me manques, et notre enfant aussi! Il paraît qu'à la guerre de pauvres soldats amputés continuent d'éprouver des douleurs dans leur jambe perdue.» (A, p. 64-65) Il explique longuement à son épouse qu'il ne se sent plus capable de composer et que l'aggravation de son état à elle l'accable de chagrin. Si toutefois il existe une possibilité de salut pour lui, écrit-il, «elle réside dans ce que je suis venu faire à Saint-Aldor. [... Cela constitue ma dernière chance. » (A, p. 67-68) Lors de la scène du pardon avec Julia, Bapaume lui apprend qu'avec sa femme et son fils, décédé au printemps passé, ils vivaient dans la pauvreté, que la tuberculose de leur fils les a ruinés, qu'il a cru que, du chagrin de la mort de son fils, il pourrait tirer l'inspiration pour composer à nouveau, mais en vain. Il assure aussi Julia qu'ils formaient une famille «heureuse, amoureuse d'ellemême» (A, p. 104). Avant de s'en aller, Bapaume écrit à Hurtubise une lettre où il lui explique que leurs deux Françoise ne pourront se rencontrer puisque son épouse est décédée. Elle ne s'est jamais remise de la mort de leur fils. Il décrit au lieutenant comment il a assisté ainsi à « la glaciation d'une existence» (A, p. 117), formulation mi-littérale, mi-figurée où s'exprime le destin à la fois physique et psychologique de Françoise, finalement morte de froid. Si le statut de Françoise semble dès lors un peu instable, il change plus radicalement encore avec la toute fin du texte. Hurtubise trouve dans la chambre que Bapaume a occupée la lettre de Françoise et se promet de la lui renvoyer. Une inquiétude toutefois le saisit, et il compare les deux lettres: celle de Françoise et celle que Bapaume lui a écrite. Le roman se finit sur ces mots: "Il n'y avait pas de doute possible: elles étaient de la même main.» (A, p. 122)

Patiemment, le roman a constitué à Bapaume, sous forme de souvenirs essentiellement, une vie familiale présente et passée, qui prend la forme d'un petit univers fictionnel en évolution, peuplé d'individus aux propriétés et relations diverses. Ce qu'on apprend de cet univers se déploie sur plusieurs mois, voire plusieurs années: la vie familiale à trois, puis à deux, puis seul. Mais il y a plus : cet 
univers comprend l'explication de la mission présente de Bapaume. Ce dernier vient à Saint-Aldor à la suite de la mort de son fils, espérant un quelconque salut, incapable qu'il est de composer cette musique qui pourrait sauver son épouse. Cet univers n'est donc pas sémiotiquement clos sur lui-même : il donne aussi son sens à l'action de Bapaume. Ses ramifications sont multiples et profondes, structurant toute la trajectoire narrative de Bapaume. Ses ramifications prennent aussi une forme manifeste dans l'action de Bapaume à Saint-Aldor : le musicien pense à son épouse, lui écrit, parle d'elle. Dans ces conditions, le fait d'apprendre, par la lettre de Bapaume à Hurtubise, le décès de Françoise est problématique; de cela, le lecteur peut se tirer tant bien que mal en supposant, par exemple, un travail du deuil particulier chez Bapaume, fait en partie de déni. L'édifice s'écroule toutefois avec les dernières phrases: d'abord, c'est la fiction familiale qui s'effondre; puis c'est la venue de Bapaume à Saint-Aldor pour demander pardon qui dès lors perd tout sens. Et la fiction romanesque de se faire autophage, à la faveur de cette mémoire singulière de Bapaume, qui livre un passé fort incertain.

La lecture attentive du roman révèle en effet un fonctionnement pour le moins insolite de la mémoire de Bapaume. D’une part, il oublie ce qui semble inoubliable: on a déjà mentionné le cas de la partition; on peut évoquer aussi cette «Louise» que le musicien croise dans le texte, qu'il prend pour «la toquée du village » et qui s'avère être celle auprès de qui il passait ses nuits durant tout son premier séjour à Saint-Aldor, vingt ans plus tôt. Elle n’a pas laissé la moindre trace dans la mémoire d'un Bapaume confus. D’autre part, le musicien semble construire ses souvenirs à partir du présent. Ainsi, il mentionne sa femme Françoise, la violoniste juive et d'origine parisienne, après que Hurtubise lui ait parlé de sa mère, prénommée Françoise, violoniste juive et d'origine parisienne. La première mention de son fils apparaît après que Chouinard ait découvert l'ourson de peluche. Dans ses souvenirs et dans son discours, sa femme décline à mesure qu'est raconté, dans le récit, le destin contemporain de la fille du bedeau: cette dernière, à l'ouverture du roman, a disparu en montagne; on craint ensuite qu'elle ne soit tombée dans une crevasse; puis on estime que sa vie est en grand péril hypothermique; et finalement les sauveteurs la découvrent, mais trop tard. Lorsque Bapaume apprend que la fille de bedeau est une enfant et non une femme, il tombe des nues. Et quand ensuite il évoque à nouveau son fils, c'est pour parler de sa mort. Tout se passe donc comme si la mémoire de Bapaume se construisait, à partir des éléments du présent, un passé qui devient dès lors réel pour lui: et les souvenirs se transforment au gré de l'évolution des événements présents.

La perspective dès lors se renverse, et ce qui apparaissait plus tôt comme un phénomène d'autophagie devient désormais autoengendrement fictionnel : par le biais de ce prisme qu'est Bapaume, les éléments diégétiques deviennent, sous le masque de souvenirs jamais vécus, matière à de nouvelles fictions en constante 
évolution. Ce qui se donnait pour mémoire se révèle machine à fictions prenant prétexte de ce qui est pour le réfracter en univers multiples. La mémoire est, littéralement, acte créateur.

Il faut alors noter, comme chez Gervais, la présence massive de l'écrit dans le roman. C'est une lettre que Bapaume «reçoit» de «Françoise», c'en est une autre qu'il lui écrit, une autre encore qu'il écrit à Hurtubise. Il écrit encore un petit fragment en pensant à Maurice. Lorsqu'à cela s'ajoute la caractéristique, peut-être métafictionnelle, de compositeur ${ }^{16}$ dont Bapaume est pourvu, on en vient à pouvoir lire, en filigrane, cette figure littéraire comme, aussi, une figure du créateur artistique - et plus précisément comme une figure du romancier. Le roman se laisse alors également penser comme une fable sur la fiction, ses origines, ses (en)jeux.

Si Lacquittement donne l'impression de vouloir déjouer le lecteur, ce n'est pas pour le seul plaisir de le prendre à revers. C'est aussi, sinon surtout, pour lui dire, à sa manière, cette pensée de la fiction : qu'elle est d'abord multiplication de mondes qui entretiennent des rapports variables entre eux, et où la cohérence n'est pas le maître mot; que les frontières logiques n'ont pas pour elle valeur d'obligation; qu'elle va, par principe, opposer à l'unicité d'un espace délimité et réglementé le savoir de ses désordres et la subversion de ses charmes ${ }^{17}$. La prolifération des incompatibilités est non pas tant une manière de refuser de se situer dans l'espace discursif de la fiction romanesque qu'une façon de revendiquer une authenticité première de la fiction, qu'une façon de resituer ses enjeux: autour d'une ligne de partage non pas entre le réel et l'imaginaire, le vrai et le faux, mais entre une unicité cohérente qui est au mieux un amoindrissement consenti, au pire un aveuglement, et une pluralité première assumée dans toute sa jubilatoire luxuriance.

16. On pourrait y voir ce genre de mise en abyme de l'énonciation dont parle Dällenbach - représentation, donc de l'énonciation romanesque. Et peut-être faudrait-il alors lire ce qui se dit de la musique dans le roman comme une mise en abyme du code, ou encore une métaphore d'origine. Voir Lucien Dällenbach, Le récit spéculaire. Essai sur la mise en abyme, Paris, Seuil, coll. «Poétique», 1977, p. 100-138. On sait par ailleurs l'importance déterminante qu'a, dans l'œuvre de Soucy en général, la question de l'engendrement - de fiction comme d'être (celui-ci servant à penser celui-là).

17. Sur la valeur subversive du roman de Soucy, on lira avec profit: Viviane Asselin, «L'acquittement de Gaétan Soucy balbutie-t-il? », dans Mélanie Carrier et Maude Poissant (dirs.), Carrefour de lectures littéraires, États de la jeune recherche littéraire, Québec, CRILCQ, coll. «Interlignes », p. 97-114. 


\section{CONCLUSION}

C'est un rapport complexe au jeu, et plus exactement aux règles, que manifestent en concordance les deux imaginaires de la fiction dont on a suivi la trace dans Les failles de l'Amérique et L'acquittement. D'un côté, et de prime abord, ces romans se font fictions ludiques, joueuses ${ }^{18}$, qui jouent avec les codes établis de la représentation romanesque pour mieux déjouer l'activité lecturale. Art de la feinte pour elle-même, en somme, volonté de rupture avec la norme - dont on sait au demeurant qu'elle est, depuis la fin du XVIII ${ }^{\mathrm{e}}$ siècle et l'avènement du romantisme, la marque de la «vraie littérature». En plus de tendre un miroir au lecteur pour qu'il saisisse, au moment même où ils se dérobent, les paramètres conventionnels de l'appréhension de la fiction romanesque, les deux romans problématisent, au moyen de leurs dispositifs ambigus, la distinction entre réalité et fiction ou imaginaire ${ }^{19}$. On le voit, c'est un usage particulier du terme jeu qu'on met à contribution ici: il ne s'agit pas d'une activité régie par des règles, mais plutôt d'une activité dont le but est de détourner les règles déjà établies. Il faudrait peut-être plus précisément parler de fictions critiques ${ }^{20}$, qui cherchent à exhiber, dans le geste même de leur détournement, des conventions qui finissaient par masquer leur nature arbitraire.

Nous avons toutefois cherché à y voir plus qu'une position dénonciatrice, en voulant lire ces romans comme l'affirmation d'une pensée, d'une conception, d'un imaginaire de la fiction romanesque. Nous avons formulé cette hypothèse que les deux romans s'attaquent à certaines conventions non par principe, mais

18. On emprunte l'expression «fictions joueuses » à Bruno Blanckeman, Les fictions singulières. Étude sur le roman français contemporain, Paris, Prétexte éditeur, 2002, p. 59110. Blanckeman fait des fictions joueuses une catégorie susceptible de décrire une ample portion de la littérature romanesque française depuis 1980.

19. Les romans pourraient alors être qualifiés de métafictions, dans le sens que donnent à ce terme Molino et Lafhail-Molino, en parlant des récits postmodernes: «la fiction devient métafiction, c'est-à-dire récit dans lequel [est intégrée] la réflexion sur le statut de la fiction et ses rapports avec la réalité». (Jean Molino et Raphaël Lafhail-Molino, Homo fabulator. Théorie et analyse du récit, Montréal, Arles, Leméac, Actes Sud, 2003, p. $\left.337-33^{8}\right)$

20. On emprunte cette expression à Dominique Viart, qui en fait un trait dominant de la production littéraire française contemporaine. Voir Dominique Viart, «Le moment critique de la littérature. Comment penser la littérature contemporaine? », dans Bruno Blanckeman, Jean-Christophe Millois (dirs.), Le roman français aujourd'hui. Transformations, perceptions, mythologies, Paris, Prétexte éditeur, 2004, p. 11-35 (et plus spécifiquement p. 29-35). 
parce qu'elles cachent la nature du discours fictionnel en s'y substituant ${ }^{21}$. Ils ont ainsi cette ambition de dire quelque chose de la fiction, d'en révéler certaines propriétés. Et, bien qu'elle ne s'y résume certes pas, la fiction est assurément création de mondes qui vont entretenir, entre eux et avec le(s) nôtre(s), des rapports multiples, ambivalents, changeants ${ }^{22}$. Ce que, surtout, ces romans nous disent, c'est le caractère exagérément réducteur de l'opération qui voudrait imposer à la fiction un principe de cohérence. Le principe de cohérence est un choix poétique, une règle normative, et non le fondement de la fiction: ce dernier, sans s'y limiter, est d'abord, et tout différemment, une mise sur pied d'univers, peuplé(s) d'objets dotés de propriétés et entretenant entre eux diverses relations. Reste que, sur le plan des règles normatives, ces romans semblent se savoir marginaux, comme le suggère leur insistance à mettre en scène des personnages au psychisme lui-même marginal.

Lambiguïté sémantique du terme « jeu» sur laquelle nous concluons - jeu avec les règles ou jeu réglé - dit peut-être aussi, à sa manière, la position ambiguë, mais dans le même temps constitutive de la littérature contemporaine: consciente, désormais, de la conventionalité de toute pratique langagière et de l'institution littéraire où elle prend forcément place; obligée, sous peine de passer pour naïve, de manifester cette conscience dans une attitude réflexive; désireuse, aussi, enfin, de compenser cette posture de subordination critique par une volonté d'affirmation, par l'instauration d'une voix qui ne serait pas que d'écho ironique.

21. On pourrait reprendre ici, pour représenter l'effort de pensée de ces romans, la distinction searlienne entre règles normatives et règles constitutives. Pour Searle, «les règles normatives gouvernent des formes de comportement préexistantes ou existant de façon indépendante; les règles de politesse, par exemple, gouvernent les relations interpersonnelles qui existent indépendamment des règles. Mais les règles constitutives, elles, n’ont pas une fonction purement normative, elles créent ou définissent de nouvelles formes de comportement. Les règles du football ou du jeu d'échecs, par exemple, ne disent pas seulement comment on joue aux échecs ou au football, mais elles créent pour ainsi dire la possibilité d'y jouer. [...] Les règles normatives ont pour fonction de régir une activité pré-existante, une activité dont l'existence est logiquement indépendante des règles. Les règles constitutives fondent (et régissent également) une activité dont l'existence dépend logiquement de ces règles» (John R. Searle, Les actes de langage. Essai de philosophie du langage, trad. Hélène Pauchard, Paris, Hermann, coll. «Savoir», 1972, p. 72-73). Tout se passe en fait comme si les deux romans cherchaient à montrer que la cohérence relève des règles normatives de la fiction, et qu'ils voulaient, tout différemment, mettre en relief ce qu'ils considéraient comme des règles constitutives.

22. Il faut relire ici les réflexions de Pavel sur les univers saillants, où il donne un aperçu de la variabilité de ces rapports. Voir Thomas Pavel, Univers de la fiction, Paris, Seuil, coll. «Poétique », 1988, p. 59-94. 